\section{Modeling Complex Living Systems: A Kinetic Theory and Stochastic Game Approach}

Nicola Bellomo

Birkhäuser Verlag Ag, 2008. 220pp. £66.99, Hardback ISBN: 978-0817645106

Journal of the Operational Research Society (2013) 64, 1866. doi:10.1057/jors.2013.124

Bellomo describes in this book the methods and findings of many years of work on modelling living systems using stochastic methods. The model is built upon a statistical physics model of a kinetic gas of particles moving within a defined volume, but instead of inert pieces of matter the particles are agents, whose dynamics 'follow rules determined by some organized, or even intelligent, ability'.

The kinetic model is developed in Chapters 2-4. It defines the state of each particle in terms of its position, velocity and activity. Macroscopic properties of the population, such as density, translational energy and size, may be calculated by appropriate integrations over the distribution functions. The number of particles in the population may not remain constant due to births and deaths. Interactions between particles are assumed to be binary, and are of two main types:

- short-range interactions, when two particles make a close encounter;

- long-range mean field interactions, when the test particle interacts with the mean field, averaged over all particles lying within the long-range action domain.

Interactions may be either conservative, resulting in no change to the number of particles, or non-conservative, leading to the proliferation or destruction of particles. To model these interactions, we need to develop the proliferation (or destruction) function, the encounter rate and the transition probability density, which models the probability of a test particle moving into a different state following an encounter with another particle in a given state. The notion of state leads to the partitioning of the population into distinct sub-populations, defined by the particles' distinct states.

Bellomo develops this basic modelling framework with several extensions to model living agents that encounter one another by moving through space, interacting by close encounters or through the effect of the mean field on individual agents.

The second half of the book develops models of four examples of intelligent agents. The first example attempts to model the social state of the agents, which ranges in $H$ discrete steps from extreme poverty to the highest social state. Interactions between agents modify their state, according to $H \times H$ matrices that describe the transitions caused by interactions between particles. The interaction matrices can model different social patterns, such as competitive or altruistic behaviour. Simulations based on these assumptions claim to show how a society's total wealth, and its distribution, could be affected by these behaviours.

Chapter 6 considers vehicle flow, where the agent is the driver of the vehicle, who may be an inexperienced driver in a slow vehicle ranging through to an experienced driver in a fast vehicle. Vehicles may move in separate lanes, with overtaking permitted. Many behaviours of drivers, as they encounter a slower or faster vehicle, can be modelled with many possibilities yet unexplored.

Chapter 7 considers the mutations and immune competition of biological cells, such as tumours. Chapter 8 discusses the modelling of crowds and swarms, in congested and panic flows. These chapters are both quite short, presenting possible models and research directions, rather than the analysis of specific models.

The concluding chapter reflects on the strengths and weaknesses of these models, pondering whether it might be possible to find mathematical approaches that could produce the same answers more quickly, whether triple interactions should be considered rather than just binary, even modelling complex psychological processes between pairs and triples of agents. Bellomo recognizes that living systems are just very difficult to model mathematically - they have purpose and intention, they need to be considered at many scales, there is no invariance principle, there may be very many different kinds of agents and so on.

The kinetic theory approach to modelling living systems is a synthetic approach, in that it sets up a model and then tries to find values of the model's parameters that produce behaviour similar to that found in nature. However, in choosing to apply a kinetic approach to modelling complex social systems, one needs to decide carefully whether this is an adequate and appropriate approach to modelling such systems. Recent work on complex agent-based dynamic networks suggests a different approach, which includes models of the social networks that already link agents together and that lead to social norms that can be very significant drivers of behaviour, perhaps more plausibly than random encounters. Recent research on social networks, thanks to the recent availability of data that was not available at the time this book was written (2008), has given new insights into social behaviour that make the use of kinetic models to simulate social systems seem already curiously old-fashioned. Certainly, kinetic models will continue to have their place in modelling cells, for example, but probably not for modelling complex, intelligent, social agents.

University of Oxford

Janet Smart 